\section{Ocean acidification could cost the UK's shellfish industry $£ 6$ billion}

Shellfish harvesting accounts for over $1 / 3$ of total fisheries landings by value in the United Kingdom. Contributing over $£ 400$ million each year, shellfish aquaculture and the wildcapture shellfish, mollusk, and crustacean fisheries are important economic drivers in the UK. Ocean acidification could affect some of these species, affecting their survival as some of their shells could be affected by low pH conditions. To understand the monetary effects of ocean acidification, the authors calculated the costs of lost shellfish harvesting on the UK economy.

The authors gathered data on consumption and the gross value added to the economy for each of the following sectors of the UK shellfish industry: cockles, crabs, lobsters, mussels, Nephrops ("Norway lobster"), scallops, shrimps, prawns and whelks. Ocean acidification projections specific to the British Isles were used to determine future $\mathrm{pH}$ levels under both medium and high-emission scenarios. After examining the published research to determine the life-history effects ocean acidification will have on these species, the authors were able to model potential harvest losses.

Vulnerability of each catch sector to the economy was determined by calculating the ratio of shellfish value to the total value of fish and shellfish harvesting. Results indicated that the scallop and Nephrops fisheries will be impacted the most. England and Wales were found to be most vulnerable in their mollusk harvesting, while Northern Ireland and Scotland were more vulnerable in their production of crustaceans. England's vulnerability was determined to be $50 \%$, Wales at $43 \%$, Northern Ireland at $79 \%$, and Scotland at $66 \%$. "Wales will potentially experience the highest ocean acidification impacts as $94 \%$ of its fisheries production is shellfish."

Economic losses were measured in two ways: net present value and partial equilibrium. Net present value essentially shows the effects ocean acidification will have from a microeconomic standpoint - just looking at the expected losses to the industry in the future from reduced harvest - whereas the partial equilibrium approach takes a more macroeconomic outlook, examining the effects to the wider economy due to the lesser supply of shellfish.

The net present value for molluscs was calculated at $£ 1,847$ million, and $£ 4,265$ million for crustaceans. Economic losses due to ocean acidification were calculated at between $£ 739-1,478$ million for molluscs and £1,279-2,559 for crustaceans, with the higher price reflecting the higher emissions scenario. Looking from a macroeconomic standpoint, the average net total loss to the economy from mollusk production was calculated at $£ 38 \pm$ 29 million, and $£ 88 \pm 47$ million for crustacean production. Combined, these losses would equate to between $£ 3$ and $£ 6$ billion of the UK's GDP in 2013.

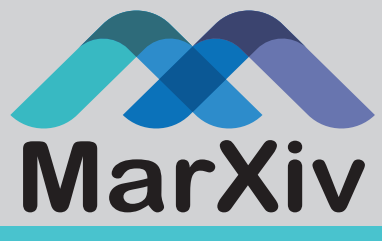

This is a summary of: The economic impacts of ocean acidification on shellfish fisheries and aquaculture in the United Kingdom

Accessible at: https://marxiv.org/d4wh7

Authors: Stephen Mangi, Jeo Lee, John Pinnegar, Robin Law, Emmanouil Tyllianakis, Silvana Birchenough

Added to MarXiv: May 2018

Published: Environmental Science \& Policy, 2018

Suggested Citation: Ocean acidification could cost the UK's shellfish industry $£ 6$ billion. OCTO (2018). DOI: 10.17605/OSF IO/4HU5R

See more MarXiv summaries at https://www.marxivinfo.org/ summaries

Join the MarXiv Summaries monthly newsletter at https:// oct.to/marxivsum

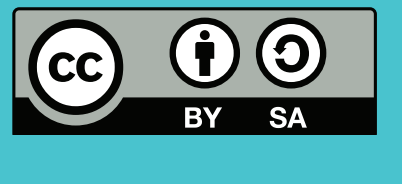

MarXiv is an Осто Initiative

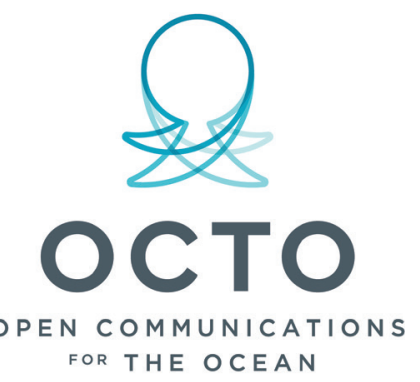

